\title{
Load Forecasting via Detrending and Deseasoning
}

\author{
Branislav Vuksanovic \\ School of Engineering \\ University of Portsmouth \\ Portsmouth, UK \\ e-mail: branislav.vuksanovic@ port.ac.uk
}

\author{
Pedro Martín \\ Department of Electronics \\ University of Alcala de Henares \\ Alcalá de Henares, Spain \\ e-mail: pedro.martin@uah.es
}

\begin{abstract}
Load forecasting is a term usually applied to describe a process of estimation or prediction of future energy demand for a certain distribution grid or part of a grid. Large number of different methods and techniques used for load forecasting have been developed in the past and new and improved methods are regularly being reported in research literature. This paper describes one of traditional load forecasting approaches based on autoregressive moving average (ARMA) modelling of load demand time-series (TS). However, it reconsiders each step in this process and proposes some new procedures to improve and clarify the whole method. Effectives of described approach is demonstrated using energy consumption measurements recently recorded at substations in central London area.
\end{abstract}

Keywords-load forecasting; stochastic modelling; autoregressive moving average model; trend; seasonality

\section{INTRODUCTION}

Load forecasting is the process of estimating future electric loads at various points in the power system ahead of actual load occurrence. Terms such are load estimation or prediction are sometimes used in the same context. Due to the changing nature of the grid operation today, the load forecasting tasks are becoming ever more important in planning the integration of distributed generation into the system. With the unbundling and opening of the energy sector to different suppliers and operators, the load forecasting is rapidly becoming important to all involved parties - energy providers, grid operators, traders and brokers since despite being responsible for different aspects of grid operation they all need load forecasting to facilitate and support their role in the process of reliable and efficient energy supply to end users.

Usually, historical load data are used to perform forecasting but several forecasting techniques can also make use of other information, e.g. historical and forecasted weather data, to aid forecasting and predict the load more accurately. Due to nature of the forecasting problem, load forecasting is usually classified as short, medium and long term load prediction problem. Short-term forecasting is used when an information about likely load in the next couple of hours up to a day period is required while a medium-term forecasting tries to predict the load for a few days and up to a few weeks' period. As such, short-term planning is used to aid allocation of spinning reserve and unit commitment while the medium-term forecasting can be used to plan for seasonal peaks as well as maintenance scheduling. Longterm forecasting tries to predict load requirements in the period of few months to a few years and more often than other two types of load forecasting includes and incorporates other factors and socioeconomic variables such are population and population growth of an area as well as gross national product in the forecast process.

Large number of forecasting techniques have been reported in the literature and used by the power companies in the past. Detailed review of the most popular techniques is presented in [1] including traditional methods such are time series, regression, econometric models as well as load decomposition techniques and models. Soft computing and artificial intelligence based techniques such are artificial neural networks (ANNs), genetic algorithms and fuzzy logic prediction techniques have also been discussed in this work. [2] reports on most popular forecasting methods in the recent decade. This work not only reviews most popular techniques and estimation algorithms but details the increase in the number of research works published in this area indicating the importance of the load forecasting problem. General conclusion is that although the number of techniques recently reported in research literature have a very good level of forecasting accuracy, there is no universal method that can outperform all other methods in all situations. Each proposed technique while having significant advantages usually has some theoretical limitations and can usually be improved by combining it with some other method through so called hybridisation process of various models. Choosing a forecasting technique is therefore a difficult task itself as due to the nature of load demand variations in one area or country one particular method can be superior to another, so understanding different techniques and their advantages and disadvantages is important.

\section{TIME SERIES PRE-PROCESSING FOR FORECASTING}

In this study, we analyse load data measured in the number of secondary transformer substations in central London. Observed substations were fed from five primary substations, where each of five primary substations fed app 70 of the secondary substations. Daily load profiles - power demand by each substation were recorded during the period of two years (2010-2012). Each daily load profile consists of 24, 48 or in some cases 96 measurements depending on the measurement frequency which can be 1 hour, $1 / 2$ hour or 15 minutes respectively. 
Stationarity is an important TS property when it comes to forecasting as most of the TS models work on the assumption that the TS is stationary. The theories related to stationary series are more mature and easier to implement compared to non-stationary TS analysis techniques. Stationarity is defined using very strict criterion, however, for practical purposes we can assume the series to be stationary if it has constant statistical properties over time, i.e. constant mean, constant variance, an autocovariance that does not depend on time (but obviously, does depend on time lag between the original and shifted signal, i.e. TS). Trend and seasonality are two major reasons behind nonstationarity of a TS recorded in distribution grid and in many other branches of industry.

Time series (TS) from our data set -30 successive load profiles measured at one of observed transformer stations in 1 hour intervals is shown in Fig. 1. To initially assess the statistical nature of this time series, rolling mean and standard deviation have been calculated and plotted on the same figure using a sliding data window of 24 measurement points which corresponds to 1 day wide data window. Even for such a relatively large interval, both characteristics seem to vary significantly over the measured period, thus the TS can be considered as non-stationary.

In this work, we adopt a classic approach of making TS stationary by modelling and removing first the trend and then seasonality components from considered TS. The forecasting is then implemented on resulting, stationary TS and the forecasted values are finally converted into the original scale by applying trend and seasonality constraints back. Several simple methods exist to estimate trends - taking average for a certain, suitable period such as monthly or weekly average (aggregation), taking rolling averages over a longer period of time (smoothing) or polynomial fitting where a regression model can be fit into TS. In this work, we apply a smoothness priors based detrending originally developed and demonstrated for removing trends from ECG signals [3]. This method operates like time-varying finite impulse response (FIR) high pass filter.

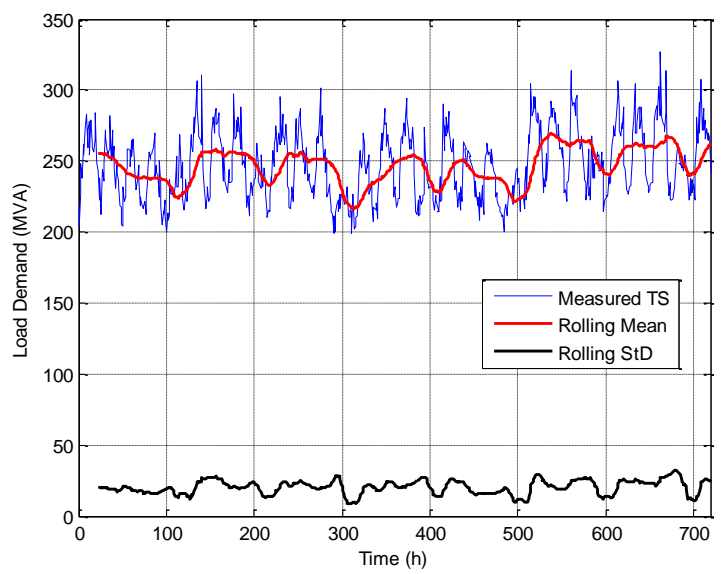

Figure 1. Measured load demand data time series with rolling statistics.

The trend component is modelled using a linear observation model and the regression parameters of the model estimated using regularised least squares fitting method. Regularisation parameter of the model $-\lambda$ is the only adjustable parameter in the optimization process, and the whole method to extract trend " $x t$ " from the measured TS " $x$ " can be summarised using Matlab code given below.

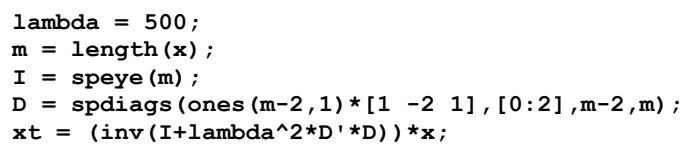

Fig. 2 shows the result of detrending using the above approach and regularisation parameter $\lambda=500$.

Frequency spectrum of detrended part of the signal is shown in Fig. 3. Four peaks in the spectrum have been detected and indicated in this figure. It is worth noting that the peak frequencies seem to be multiples of the lowest detected frequency of 0.083 or 12 samples per period, since the frequency resolution of the spectrum might slightly obscure this fact.

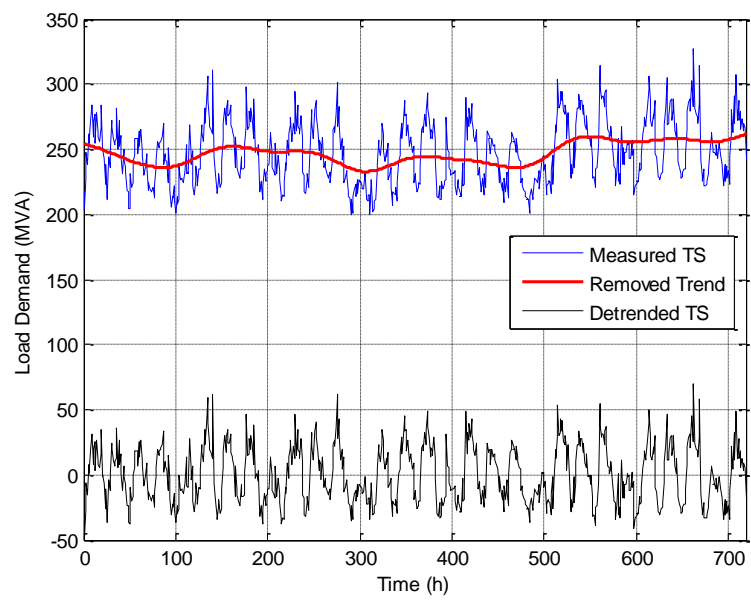

Figure 2. Estimated trend and residual, detrended load data.

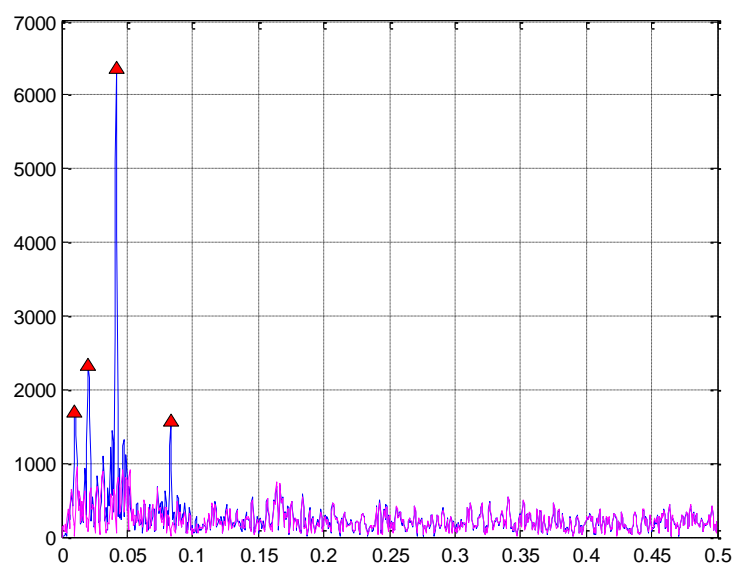

Figure 3. Frequency spectrum of detrended load signal.

Thus, the spectral analysis reveals seasonality corresponding to periods of 12, 24, 48 and 96 samples in TS. 
However, the most prominent peak confirms the expected daily seasonality of TS, i.e. it corresponds to frequency of 0.0417 or $1 / 0.0417=24$ samples per period. Since the time between two samples is 1 hour, this corresponds to daily seasonality in TS. Using the method of least squares fitting [4] we can now remove this component from the signal. TS with removed seasonality is shown in Fig. 4 and the spectrum of this TS is added to Fig. 3.

Removal of one seasonality component corresponding to normalised frequency " $\mathrm{n} n$ " identified in Table I from-TS and indicated in Fig. 3 can be accomplished using the following piece of code:

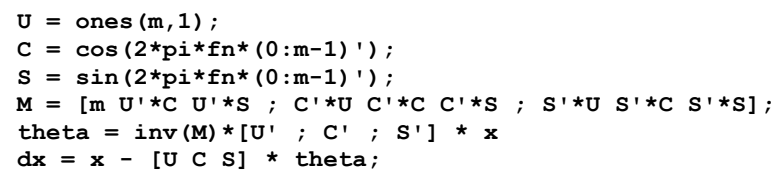

Rolling mean and standard deviation characteristics are again calculated and plotted on the same figure indicating significantly improved stationarity of the obtained TS.

Those simple trend reduction techniques might not work well in all cases, particularly the ones with high seasonality. Sometimes, differencing - taking the difference of a given TS with a particular time lag can be used to more effectively remove both trend and seasonality.

In this work, we first remove both trend and seasonality from the original, measured TS and follow this by differencing the remaining signal to ensure even stronger stationarity of the residual TS. Differencing of any order can be implemented using "diff" function, or alternatively, to take the first difference of TS " $x$ " we can use the following instruction in Matlab:

$\mathrm{d} \mathbf{x}=\mathbf{x}-[0 ; \mathbf{x}(1:$ end -1$)]$

Result - detrended, deseasoned and differentiated TS is shown in Fig. 5.

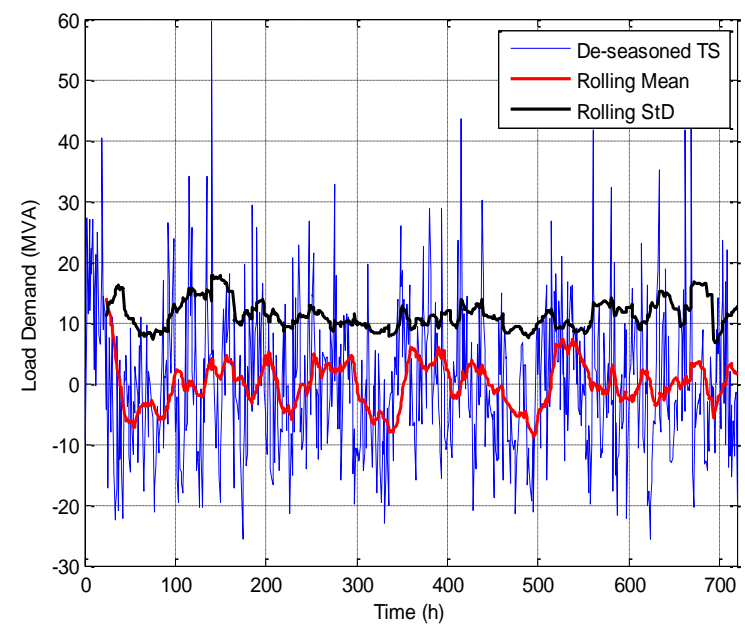

Figure 4. Detrended and de-seasonalised load data.

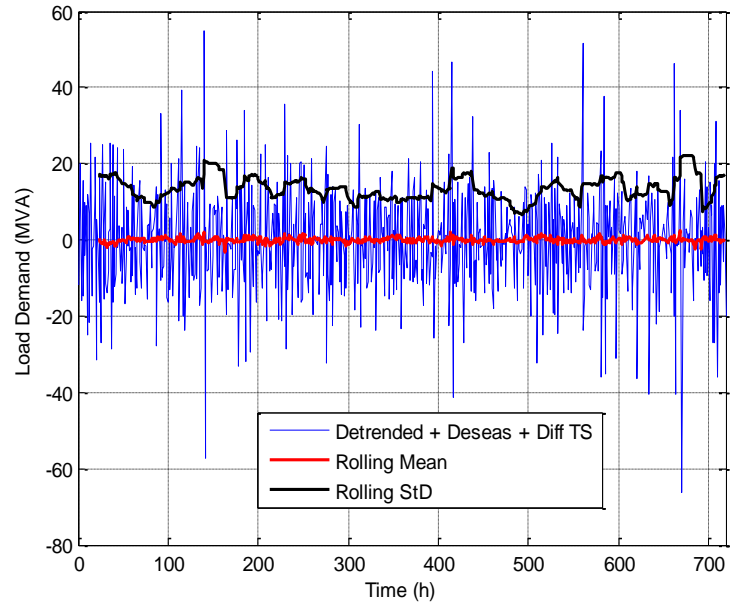

Figure 5. Detrended, de-seasonalised and differenced load data.

\section{FORECASTING USING ARMA MODELS}

Having obtained stationary TS we can now approach the task of estimating the underlying autoregressive-moving average (ARMA) model of this TS.

ARMA model combines two submodels - autoregressive (AR) and moving average (MA) models, i.e. it includes lagged and weighted terms of the signal itself as well as weighted noise or residual terms. ARMA model combining autoregressive model of order $\mathrm{p}$ and the moving average model of order $\mathrm{q}$ is usually denoted as ARMA (p,q) and mathematically described as:

$$
x[n]=\sum_{k=0}^{q} b_{k} w[n-k]+\sum_{k=1}^{p} a_{k} x[n-k]
$$

The order of two sums in (1) is not important as both AR and MA models are of linear and time-invariant type and can be interchanged.

Signal modelling using this approach is a two-step process. We first need to decide on the actual model type AR, MA or ARMA and then determine the order(s) of the selected model type. The second step in this process is to estimate the coefficients of selected model. Coefficients of AR type model can be obtained using a least-squares regression while the estimation of parameters for MA and ARMA models usually requires a more complicated iteration procedure [5]. Usually a computer program is used to implement this procedure with little or no user interaction.

One of most common approaches used to determine the orders $p$ and $q$ of $\operatorname{ARMA}(p, q)$ model is via inspection of the autocorrelation function (ACF) and partial autocorrelation function (PACF) characteristics, described by [6]. ACF at lag $\mathrm{k}$ measures the correlation of the signal or time series with itself shifted $\mathrm{k}$ samples, PACF at lag $\mathrm{k}$ is obtained from the autocorrelation at lag $\mathrm{k}$ after removing autocorrelation with a $\mathrm{AR}(\mathrm{k}-1)$ model. If the $\mathrm{AR}(\mathrm{k}-1)$ model effectively whitens the signal, the PACF at lag $\mathrm{k}$ is zero. Determining the order of ARMA model via analysis of ACF and PACF characteristics 
is not a straight forward task and can require some experience, especially if higher order ARMA models are to be used. Usually, PACF plot suggests the required order of MA part of the model and any spikes at particular lag $\mathrm{k}$ in PCAF indicate the significance of the moving average coefficients at that position. ACF characteristic is used to identify the properties and the required order of the autoregressive section of the ARMA model. The lag beyond which the PACF falls permanently below the $95 \%$ or $\pm 2 / \sqrt{N}$ confidence interval indicates the number of required AR terms in the model. On the other side, high values in the $\mathrm{ACF}$ plot at regular and fixed intervals usually indicate presence of seasonality in the signal but if the ACF plot does not decay below the confidence interval, signal to be modelled is usually non-stationary. Difference of the signal should in that case be taken before any model estimation takes place.

We use ACF and PACF plots to estimate the order of the ARMA model to be used for modelling our TS. Those plots can be easily produced in Matlab using "autocorr" and "parcorr" functions. Both characteristics are shown in Fig. 6.

Considering confidence bounds - horizontal limits indicated in those plots, the only significant value in ACF function is at the first lag, whilst PACF charcateristics contains significant values at lags 1 to 5 and at lags number 10 and 11. Thus although we can use both $\operatorname{ARMA}(11,1)$ and $\operatorname{ARMA}(5,1)$ models to model this TS, we opt for a simpler model with no moving average section, i.e. we perform the modelling and show results achieved using $\operatorname{ARMA}(5,0)$ model which is sometimes simply referred to as $\operatorname{AR}(5)$ model as it contains no MA section.

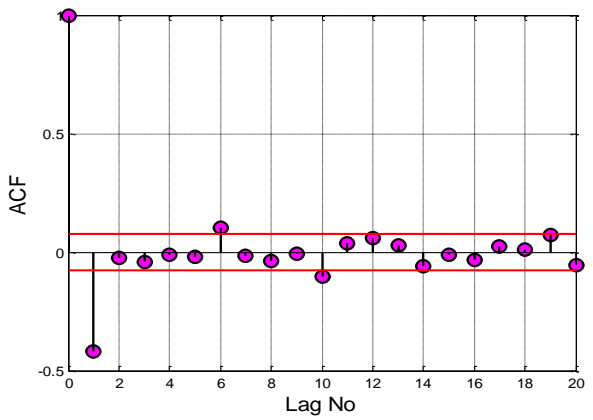

a)

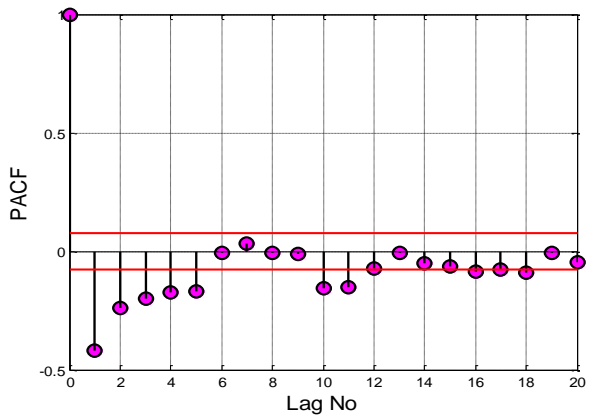

b)

Figure 6. a) ACF and b) PACF characteristics for analysed TS.
Various techniques to estimate ARMA and AR models can be found in literature and have been implemented in numerous packages and functions. Usually, coefficients of AR model are obtained by solving Yule Walker set of equations [7] which relates those coefficients to autocovariance of considered TS. Matlab function "aryule" provides an efficient way to solve those equations and obtain a resulting set of coefficients using a well-known Levinson recursion [7].

Thus the coefficients of the AR(5) model can be obtained in Matlab using:

$[d, p]=\operatorname{aryule}(x, 5)$

After this instruction, AR coefficients will be contained in a 6 elements row vector " $p$ " with the leading unity coefficient followed by five AR coefficients calculated using Levinson recursion method.

Parameter " $d$ " contains the required noise variance for the white noise input to this model in the forecasting phase. Forecasting is accomplished using "filter" function in Matlab in the following way:

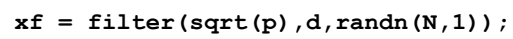

Having predicted differenced version of detrended and deseasoned TS, the applied pre-processing needs to be "reversed" in order to generate total forecast for the original, measured TS. Predicted set of measurements is first "integrated" to account for differencing of TS immediately before prediction. This step can be accomplished in Matlab using "cumsum" function in the following way:

$x f=[x(e n d) ; x(e n d)+\operatorname{cumsum}(x f)] ;$

Here, " $x(e n d)$ " represents the last element of the original, undifferenced TS which is needed to reconstruct the values of predicted set to its proper level. This element is then also used as a first element of the forecasted set. This is an optional step used here, for no other reason but to connect existing - measured data points with the forecasted set during the plotting of results.

\section{FORECASTING TREND COMPONENT OF TS}

Once the random part of TS has been generated, the seasonal and trend components need to be forecasted and added to it to obtain the full forecast. Seasonal component is deterministic in nature since its frequency fn is known, as it has been determined earlier in the pre-processing stage, and the amplitude as well as phase are easily determined. Thus, the forecasting of seasonal component is a straight forward task and does not introduce significant error in the final forecast.

\section{A. Trend Forecasting Using Multiple Linear Regression (MLR)}

Trend component, although nonlinear in nature can, somehow surprisingly be predicted using linear regression 
(LR) technique. Linear regression [8] assumes linear model of type:

$$
x=\beta_{0}+\beta_{1} t
$$

where $x$ is the dependent variable, $t$ is independent variable and $\beta_{0}$ and $\beta_{1}$ are parameters of the model. This model can be expended to so called multiple linear regression (MLR) model which is a multivariate version of linear regression and can therefore be applied to $m$ independent variables $t_{1}, t_{2}$, $\ldots, t_{m}$ in the form:

$$
x=\beta_{0}+\beta_{1} t_{1}+\beta_{2} t_{2}+\ldots+\beta_{m} t_{m}
$$

MLR model can be estimated using " $\mathrm{f} i \mathrm{tm}$ " function in Matlab. Implementation is relatively straightforward in case of truly multivariate data set but some data preparation is needed if the same approach is to be used for the univariate TS modelling. Excerpt of Matlab code given below illustrates this approach assuming available samples of trend component have previously been saved in column vector " $\mathrm{x}$ " containing $m$ measurements (data points).

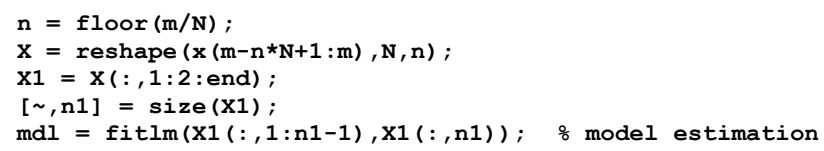

Here, a univariate time-series " $\mathrm{x}$ " (one observation per time instant) is converted into multivariate set of $n$ observations by reshaping vector " $\mathrm{x}$ " into $N \times n$ data matrix " $\mathrm{X}$ ". $\mathrm{N}$ represents number of points to be predicted and is sometimes referred to as dimensionality of multivariate data set. Columns of matrix $\mathrm{X}$ are formed by taking $\mathrm{N}$ successive measurements from the TS " $x$ ", thus the first two lines in the above code are used to ensure that only the last $N \times n$ elements of vector " $\mathrm{x}$ " are used in the process of generating data matrix " $X$ ". Odd columns from this matrix are then further extracted into matrix " $\mathrm{X} 1$ " and used to estimate MLR model "mal".

Once the model is estimated, the rest of the data - even numbered columns from matrix " $X$ " are used to obtain the $\mathrm{N}$ elements long forecast " $\mathrm{x} \mathrm{f}$ " in the following way:

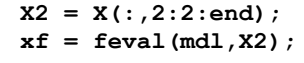

Final result, total TS forecast is obtained by adding all three predicted components - stochastic part of TS, predicted using AR approach, seasonal component produced by simply extending the deterministic seasonal component identified in the TS and a trend component, predicted using MLR model discussed in this section. Example result is shown in Fig. 7 indicating a very good agreement between the measured daily load profile and predicted points.

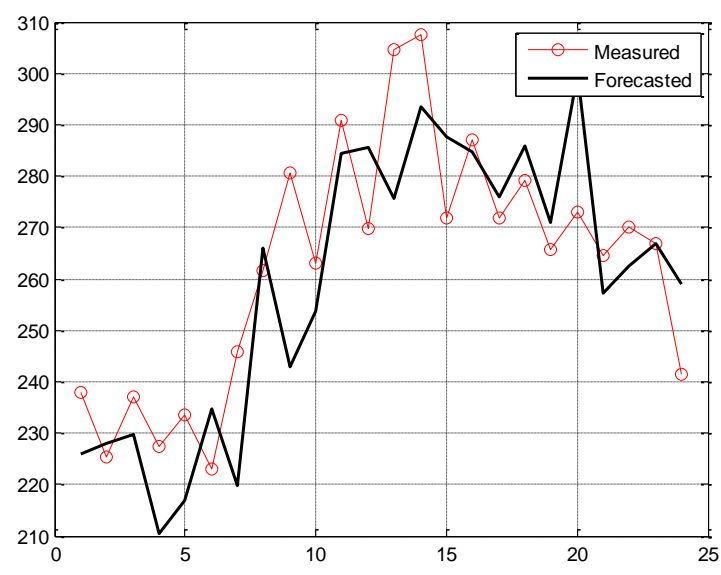

Figure 7. Measured and forecast load demand.

\section{CONCLUSIONS AND SOME DIRECTIONS FOR FURTHER WORK}

This work presents a load forecasting approach using traditional ARMA based modelling of measured TS. Although this approach is well known and studied in practice and these days somewhat outperformed using modern AI based techniques, such are artificial neural networks or support vector regressors, it is still widely used for forecasting various types of TS over short and mid-term periods. Paper proposes a new technique to decompose TS into three main components and illustrates techniques to forecast each of extracted components individually in order to finally reconstruct the full TS predicted by summing three components together in the final stage of the forecasting process.

Accuracy of the proposed method depends on a small number of parameters closely related to nature of measured TS and therefore easily analysed and adjusted. However, deeper analysis of each of those parameters on the forecast accuracy is necessary and is planned in the extension of this work.

\section{REFERENCES}

[1] L. Suganthi and A. A. Samuel, "Energy models for demand forecasting - A review," Renew. Sustain. Energy Rev., vol. 16, no. 2, pp. 1223-1240, 2012.

[2] I. Ghalehkhondabi, E. Ardjmand, G. R. Weckman, and W. A. Young, An overview of energy demand forecasting methods published in 2005-2015, no. May. 2016.

[3] P. A. Karjalainen, "An advanced detrending method with application to HRV analysis Mika P. Tarvainen, Perttu O. Ranta-aho, and Pasi A. Karjalainen," pp. 1-4.

[4] G. Blanchet and M. Charbit, Digital Signal and Image Processing using MATLAB. 2006.

[5] C. Chatfield, The Analysis of Time Series: an Introduction, vol. 140, no. 2. 2004.

[6] G. E. P. Box and G. M. Jenkins, Time series analysis: forecasting and control. 1976.

[7] Heyes, M. H. J. Gruber, and M. H. Hayes, "Statistical Digital Signal Processing and Modeling," Technometrics, vol. 39. p. 335, 1997.

[8] S. Araghinejad, Data-Driven Modeling: Using MATLAB® in Water Resources and Environmental Engineering. 2014. 